\title{
ReaR
}

ISNN 19894090

Revista electrónica de AnestesiaR

Febrero 2022

ARTÍCULOS DE REVISIÓN

\section{Aproximación a las terapias de remplazo renal continuas.}

Martínez Villar C, Charco Roca LM, Fernández Ruipérez L, Canales Lara PM

Complejo Hospitalario Universitario de Albacete.

\section{Resumen}

La insuficiencia renal aguda (IRA) se define como una disminución abrupta de la función renal y constituye una entidad muy frecuente en las unidades de cuidados intensivos (UCI). Su etiología es multifactorial y se estima que en torno a un 5-10\% de los pacientes que desarrollan IRA en las UCI van a requerir de una terapia de remplazo renal.

El objetivo de esta revisión bibliográfica es hacer una aproximación a los conocimientos que debe poseer un anestesiólogo sobre el manejo de las terapias de remplazo renal. Para la elaboración de esta revisión se ha realizado una búsqueda bibliográfica no sistemática en las bases de datos Pubmed y Cochrane y se incluyen metaanálisis, revisiones y guías de práctica clínica publicados en los últimos 10 años.

\section{Introducción}

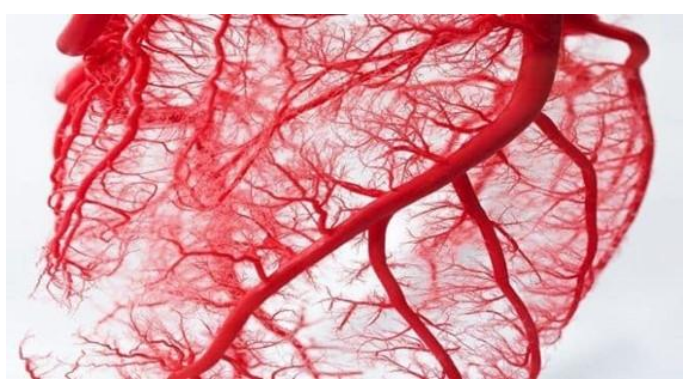

La insuficiencia renal aguda (IRA) se define como una disminución abrupta de la función renal y constituye una entidad muy frecuente en las unidades de cuidados intensivos (UCI). Su etiología es multifactorial y se estima que en torno a un 5-10\% de los pacientes que desarrollan IRA en las UCI van a requerir de una terapia de remplazo renal.

El objetivo de esta revisión bibliográfica es hacer una aproximación a los conocimientos que debe poseer un anestesiólogo sobre el manejo de las terapias de remplazo renal. Para la elaboración de esta revisión se ha realizado una búsqueda bibliográfica no sistemática en las bases de datos Pubmed y Cochrane y se incluyen metaanálisis, revisiones y guías de práctica clínica publicados en los últimos 10 años.

La insuficiencia renal aguda es una de las complicaciones más comunes en los pacientes críticos y se define como una disminución abrupta de la función renal (1).

Los criterios diagnósticos más utilizados son los establecidos según las guías de práctica clínica KDIGO (2) (Tabla 1).

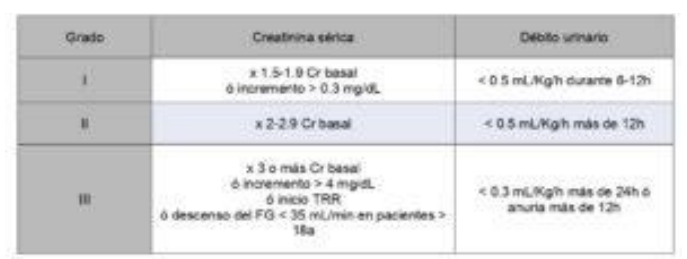

Tabla 1: Estadios de daño renal según la clasificación KDIGO (2).

Se ha establecido que la causa más frecuente de insuficiencia renal aguda en los pacientes críticos es la necrosis tubular aguda, la mayoría de los casos 
secundaria a situaciones de hipoperfusión/isquemia renal (shock, hipotensión...), pero también secundaria a nefrotoxinas exógenas (contraste intravenoso, antibióticos...) o endógenas (rabdomiolisis, hemólisis...). Como otras causas frecuentes tenemos: causas obstructivas (traumáticas $\mathrm{O}$ farmacológicas), hipertensión intraabdominal que genere un síndrome compartimental abdominal, y otras patologías sistémicas de etiología autoinmune.

Según las distintas series, se estima que en torno a un $5-10 \%$ de los pacientes críticos que presentan insuficiencia renal aguda van a requerir terapias de remplazo renal (TRR) (3). Dentro de estas técnicas, encontramos diferentes modalidades, que pueden ser intermitentes, como la hemodiálisis intermitente (HDI); híbridas, como la diálisis sostenida de baja eficiencia (SLED, del inglés sustained lowefficiency dialysis); o continuas. Estas últimas, denominadas terapias continuas de remplazo renal (TCRR), son las más ampliamente utilizadas en Unidades de Cuidados Intensivos (UCI).

Las TCRR tienen su principal utilidad en pacientes que presentan insuficiencia renal aguda asociada a inestabilidad hemodinámica, en contraposición a las técnicas intermitentes que son, por lo general, peor toleradas hemodinámicamente. Por tanto, en esta revisión nos centraremos en las TCRR y en todo lo necesario para su aplicación en pacientes críticos que lo precisen.

\section{Método}

Para la elaboración de este manuscrito se ha llevado a cabo una revisión no sistemática de artículos indexados en MEDLINE (PubMed) de los últimos diez años, con fecha de actualización diciembre 2010, utilizando las palabras clave "critically ill", "acute kidney injury", "continuous renal replacement therapy", "sepsis" y "dyalisis". Se incluyen metaanálisis, revisiones $\mathrm{y}$ guías de práctica clínica. Se excluyeron aquellos artículos realizados en población pediátrica $\mathrm{y} / \mathrm{o}$ que no estuvieran publicados en inglés 0 español.

\section{Indicaciones, fundamentos y fisiología de las TRR}

\section{Indicaciones de las terapias de} remplazo renal (TRR) (3) (4)

- Acidosis metabólica severa: $\mathrm{pH}$ $<7-7.2$ refractaria a tratamiento médico.

- Uremia severa con clínica asociada: neuropatía, miopatía, encefalopatía o pericarditis.

- Sobrecarga de volumen que no responde a diuréticos, asociado con oliguria o anuria.

- Hiperpotasemia: $>6.5 \mathrm{mmol} / \mathrm{L}$ con o sin cambios en el electrocardiograma, refractaria a tratamiento médico.

- Intoxicaciones con toxinas dializables (por ejemplo, salicilatos).

- Otros trastornos electrolíticos refractarios a tratamiento médico, o que por su gravedad precisan de corrección innmediata, por ejemplo, hipernatremia o hiponatremia severa; o hiperfosfatemia grave.

En función de la situación clínica del paciente y de la emergencia de la patología a tratar se indicará una terapia intermitente o continua.

\section{- Momento de inicio de las TRR (5)}

Son diversos los estudios que se han centrado en analizar las ventajas/desventajas de un inicio precoz vs tardío de las TRR, siendo todavía 
motivo de controversia. Para ayudarnos a decidir, sobre si debemos indicar de forma precoz o tardía la TRR, contamos con herramientas como las escalas de insuficiencia renal aguda KDIGO, RIFLE y AKIN, así como con las indicaciones absolutas clásicamente establecidas para pautar una terapia de remplazo renal.

El inicio temprano de la terapia se refiere a aquellos pacientes que presentan datos de insuficiencia renal aguda, pero que aún no han desarrollado un criterio absoluto de TRR. Sin embargo, el inicio tardío se refiere a aquellos pacientes en los que alguna indicación absoluta de las mencionadas ya está establecida.

Los datos obtenidos de los diferentes estudios observacionales son contradictorios. En algunos se ha observado que el inicio temprano de la TRR puede reducir la mortalidad en grupos seleccionados. Sin embargo, los resultados obtenidos sobre la recuperación de la función renal dependiendo del momento de inicio de la TRR son muy variables, y algunos sugieren que no hay asociación.

En ensayos controlados randomizados [MOU1], no se han visto diferencias de mortalidad significativas. De hecho, en dos ensayos con gran número de pacientes (6)(7) se llegó a conclusiones contrarias.

En una revisión realizada por la Cochrane (8), se concreta que el inicio precoz de las TRR puede traducirse en una mejoría de la función renal y una disminución de la mortalidad, a expensas de un aumento del riesgo de efectos adversos. Sin embargo, establecen que no se ha de emplear de forma rutinaria debido a que la evidencia científica es limitada y se carece de datos de alta calidad. Por este motivo, recomiendan que la decisión de cuándo instaurar una TRR debe basarse en las características individuales de cada paciente y en el juicio clínico del personal médico responsable.

Por último, en 2020 se publicó un ensayo clínico aleatorizado multicéntrico (9), en el que no se evidencia una disminución de la mortalidad a los 90 días en los pacientes en los que se inició de forma precoz la TRR.

En resumen, dado que la evidencia al respecto es limitada, el momento de inicio de la TRR será de forma individualizada y tomada por su equipo médico responsable.

\section{- Fundamentos físicos (10)}

La TRR se utiliza para reemplazar la función renal normal. Dependiendo de la situación clínica del paciente se va a prescribir un tipo de terapia u otra.

Es importante conocer los diferentes elementos que componen una máquina de hemofiltración, que podrán variar dependiendo del tipo y de la marca del dispositivo.

La TRR consiste por tanto en un proceso para depurar productos de desecho de la sangre, que normalmente se eliminan por el riñón. Esta separación se realiza a través de una membrana semipermeable. Las membranas están formadas con una agrupación de capilares dentro de los filtros, donde la superficie total de la membrana en cada filtro depende del número de fibras. Dependiendo de las características de rendimiento del filtro se obtienen las aplicaciones potenciales de cada membrana.

Las membranas habitualmente utilizadas en hemofiltración funcionan como cualquier membrana semipermeable del cuerpo humano. Son 
permeables de forma selectiva, presentando una elevada permeabilidad al agua y una baja resistencia al paso de solutos. Se puede considerar la membrana como la unidad funcional del hemofiltro.

\section{El coeficiente de ultraflltración de la} membrana (KUF) representa la permeabilidad al agua de la membrana, medida por unidad de presión $\mathrm{y}$ superficie. La unidad de medida es $\mathrm{ml} / \mathrm{h} / \mathrm{mmHg} / \mathrm{m}$. El KUF se utiliza para definir membranas de «alto flujo» $\mathrm{O}$ «bajo flujo».

La fracción de filtración (FF), se define como la cantidad de agua que vamos a ultrafiltrar durante el procedimiento, del total de plasma que filtramos durante una unidad de tiempo determinada; y es equivalente a la cantidad de agua plasmática que eliminamos respecto a la que reintroducimos.

Se recomienda que la FF sea menor o igual al $25 \%$. Si es muy alta, existe riesgo de coagulación del hemofiltro por acumulación de elementos formes de la sangre. Si por el contrario la FF es demasiado baja, se produce una pérdida de eficacia en la técnica.

Se debe calcular en cada paciente para adaptarla de forma individualizada, con la siguiente fórmula:

Balance $(\mathrm{ml} / \mathrm{h})+/$ - Reposición prefiltro + reposición postfiltro

$\mathrm{FF}=$

reposición prefiltro

Flujo plasmático +

Donde flujo plasmático $=$ flujo de la bomba de sangre $(\mathrm{ml} / \mathrm{min}) *$ x $(100$ hematocrito/100).

*multiplicar por 60 para pasarlo a $\mathrm{ml} / \mathrm{h}$
Donde flujo plasmático $=$ flujo de la bomba de sangre $(\mathrm{ml} / \mathrm{min}) * x(100-$ hematocrito/100).

*multiplicar por 60 para pasarlo a $\mathrm{ml} / \mathrm{h}$

Los solutos que se pretenden eliminar durante una TRR se clasifican habitualmente según su peso molecular en Daltons (tabla 2). De este modo, encontramos moléculas pequeñas, como la urea, la creatinina e iones; moléculas medianas, como la vitamina B12, la glucosa o el ácido úrico; y moléculas grandes como la albúmina y la mioglobina.

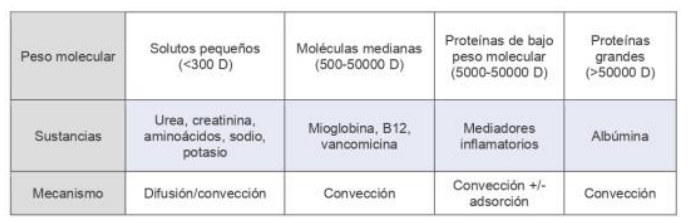

Tabla 2. Peso molecular según tipo de soluto depurable con terapia de reemplazo renal.

Por tanto, los solutos se eliminan por diferentes mecanismos dependiendo de su peso molecular y tamaño. Las moléculas pequeñas como urea, creatinina, o aminoácidos e iones, se eliminan por difusión o por convección. En cambio, las moléculas medianas y grandes, como mioglobina, vitamina B12, vancomicina o albúmina, se eliminan por convección. Para ello se utiliza la hemofiltración como veremos a continuación.

Un tipo especial son los mediadores inflamatorios, ya que son proteínas de bajo peso molecular (entre $5000 \mathrm{y}$ 50000 Daltons), que son eliminadas tanto por convección como por adsorción (11).

Los principios físicos empleados para la depuración de sustancias determinan el tipo de TRR que podemos utilizar:

- Ultrafiltración: es el movimiento de agua a través de una membrana semipermeable impulsado por un 
gradiente de presión. Se genera mediante un gradiente de presión entre la sangre y el líquido de ultrafiltrado o dializado. El gradiente de presión va a estar determinado por la presión hidrostática en sangre, la presión hidrostática en el compartimento del líquido de ultrafiltrado/dializado, y la presión oncótica de la sangre a lo largo de la fibra. La diferencia de presión total se denomina presión transmembrana o PTM, y es la que produce la ultrafiltración.

- Difusión: es el movimiento de solutos a través de una membrana semipermeable, mediante un gradiente pasivo de concentración. Los solutos se mueven desde una zona más concentrada a otra menos concentrada hasta alcanzar el equilibrio entre los dos compartimentos.

- Convección: mediante este proceso los solutos se mueven de un compartimento a otro, a través de los poros de la membrana, arrastrados por un movimiento de fluido (UF). Este movimiento de fluido se produce mediante la creación de un gradiente de presión hidrostática transmembrana, como ya se ha explicado anteriormente. Es decir, se genera una presión positiva a uno de los lados de la membrana que genera el paso de líquido, junto con solutos, desde un compartimento a otro.

A diferencia de la difusión, que como mecanismo de eliminación está limitado a solutos relativamente pequeños, con la convección se puede conseguir la eliminación de solutos de peso molecular mayor, a una tasa más alta.

- Adsorción: es un proceso extracorpóreo en el que moléculas de alto peso molecular disueltas en plasma (por ejemplo, mediadores inflamatorios) se unen a la estructura de la membrana o de otros adsorbentes (como carbón, resinas o geles). Es una técnica en crecimiento, que tiene como finalidad adsorber selectivamente moléculas que circulan por nuestro torrente sanguíneo.

- Terapias sorbentes: Se realiza mediante hemoperfusión con materiales como zeolitas y carbón. Nuevos compuestos fijados a filtros especiales han permitido mejorar su hemocompatibilidad; por ejemplo, los cartuchos con polimixina-B.

\section{- Modalidades TCRR (12).}

Las diferentes modalidades de TCRR que se utilizan en UCI están basados en los mecanismos descritos previamente, y son:

- Ultrafiltración continua lenta (SCUF): el principio físico de esta modalidad es la ultrafiltración, por la cual se va a eliminar agua plasmática del torrente sanguíneo. Este modo está principalmente indicado en pacientes con datos de sobrecarga hídrica que, o bien no responden a tratamiento médico, o queremos evitar las posibles complicaciones (alteraciones electrolíticas) que provocan las terapias diuréticas a dosis altas. El parámetro fundamental a configurar en este modo será el flujo de agua que queremos eliminar, expresado en $\mathrm{ml} / \mathrm{h}$.

- Hemofiltración veno-venosa continua (HFVVC): el principio físico de esta modalidad es la convección para la eliminación de solutos, y la ultrafiltración para eliminación de agua. Durante este procedimiento, el circuito sanguíneo recibe una cantidad de solución de reposición, la cual restituye el plasma reducido de volumen $y$ reduce la concentración de soluto. Este 
líquido de reposición se puede infundir pre filtro (predilución), o post filtro (posdilución). En función de cómo se ajusten los parámetros de reposición, se obtiene una FF. La FF no debe superar el $25 \%$ ya que fracciones superiores de filtración pueden generar problemas de hemoconcentración que acortarían la vida del filtro. También hay que tener en cuenta que, en cuanto a eliminación de solutos, la reposición posdilución es más efectiva. Dependiendo del ultrafiltrado que se quiera realizar, repondremos parcial o totalmente las pérdidas.

- Hemodiálisis veno-venosa continua (HDVVC): el principio físico de esta modalidad es la difusión. El líquido de diálisis fluye contracorriente, eliminando solutos del plasma sanguíneo por diferencia de concentración. Se ajusta el flujo del líquido dializante, también en $\mathrm{mL} / \mathrm{h}$, en función del aclaramiento que se quiera obtener. Además, se ajustará también el flujo de pérdidas que deseemos realizar.

- Hemodiafiltración venovenosa continua (HDFVVC): esta modalidad va a combinar las técnicas de difusión y convección. El líquido eliminado por convección se sustituye parcial o completamente con líquido de reposición (pre filtro o post filtro), pero además el líquido dializante va a fluir contracorriente por el compartimento de dializado. Se ajusta tanto el flujo de reposición, como de líquido dializante y las pérdidas.

\section{- ¿Qué modalidad es la más recomendada de TCRR?}

A la hora de prescribir una modalidad de TCRR tendremos que tener en cuenta numerosos factores; como la situación clínica del paciente, la indicación por la que pautamos la terapia, la posibilidad o no de anticoagular, o la susceptibilidad de desarrollar complicaciones.

Para depuración de solutos, utilizaremos HFVVC, HDFVVC o HDVVC. En pacientes con FRA no inducido por sepsis y sin contraindicaciones para la anticoagulación, parece razonable utilizar la HDVVC. Sin embargo, en pacientes con FRA y contraindicación para la anticoagulación, las técnicas convectivas presentan la ventaja de alargar la vida del filtro y evitar que se coagule el circuito mediante la reposición prefiltro. En el caso del FRA inducido por sepsis, son varios los estudios que han demostrado que no hay disminución de la mortalidad ni mejoría de la recuperación renal en los pacientes sépticos a los que se les aplica una terapia con eliminación de citoquinas proinflamatorias, ya sea por terapias convectivas como adsorbentes (13)[2]. Por lo tanto, parece que la HDFVVC presenta ventajas respecto a las demás, ya que, al combinar ambos modos, nos va a permitir la adecuada eliminación de solutos $y$ una mayor vida del filtro. (14)[3]

Para eliminación de agua del plasma sanguíneo, sin necesidad de depuración de solutos, se utilizará preferentemente SCUF.

En resumen, hay que individualizar dependiendo de cada caso, y será tarea del equipo médico elegir entre una $u$ otra modalidad.

\footnotetext{
- Accesos vasculares para TCRR en UCI (15)
} 
La colocación de un acceso vascular para iniciar una TCRR debe ser una técnica aséptica, realizada por un operador experimentado. La ecografía aumenta la seguridad y reduce el número de complicaciones asociadas.

La primera opción es la cateterización de la vena yugular derecha, seguida de la vena femoral. Si no fuera posible, la siguiente elección sería la vena yugular izquierda.

La última opción es la vena subclavia, debido a que la cateterización de esta vena puede producir una estenosis venosa central. Esto podría impedir la ulterior colocación de un acceso venoso permanente con [MOU4] fístula arteriovenosa, sí que sería necesario si el paciente precisara posteriormente hemodiálisis intermitente (HDI) de forma crónica.

\section{- Anticoagulación. (16)}

La coagulación del circuito de la máquina es un problema frecuente en las UCIs y que tiene implicaciones significativas. Supone pérdida de tiempo de terapia debido al cambio de los componentes del circuito, lo cual disminuye la eficacia del tratamiento, y alteraciones volémicas y anemización derivadas de la pérdida del contenido hemático incluida en el circuito a desechar.

Para evitar la coagulación del circuito disponemos de diversas estrategias y opciones de anticoagulación, que se dividen principalmente en cuatro grupos: $\sin$ anticogulación, anticoagulación regional, anticoagulación sistémica, y anticoagulación en casos especiales.

\section{- Sin anticoagulación.}

La terapia sin anticoagulación es la primera elección en pacientes con alto riesgo de sangrado. Esto puede venir dado por varias condiciones, entre ellas, una trombocitopenia severa, coagulopatía, disfunción o fallo hepático, así como contraindicaciones para el uso de citrato.

La vida útil del circuito, y por tanto, la duración de la terapia, puede prolongarse siguiendo las siguientes estrategias:

- Disminuyendo la hemoconcentración dentro del filtro a través de la infusión del fluido de reposición en modo prefiltro $o$ predilución $(2 / 3$ prefiltro y $1 / 3$ postfiltro).

- Manteniendo una fracción de filtración menor al $20 \%$.

- Mediante altos flujos de sangre (200-300 $\mathrm{ml} / \mathrm{min})$.

Los pacientes anticoagulados de forma crónica por otra indicación (por ejemplo, fibrilación auricular) no requieren anticoagulación adicional para TCRR.

\section{- Anticoagulación regional.}

Esta modalidad consiste en anticoagular el circuito de hemofiltración sin repercusión sistémica, permitiendo prolongar la vida útil del hemofiltro sin anticoagular al paciente. Por tanto, puede ser utilizada en pacientes con riesgo de sangrado moderado o alto.

Hay dos formas de anticoagulación regional (AR): con citrato, la primera elección; y con heparina.

- La AR con citrato es la primera opción de anticoagulación recomendada para TCRR en las guías KDIGO. Existe evidencia de ensayos controlados aleatorizados (RCTs) de menores tasas de sangrado, reducción de costes y prolongación de la vida útil de la máquina de hemofiltración. No 
obstante, la mortalidad observada es similar cuando se compara con la anticoagulación sistémica con heparina.

Se puede utilizar citrato trisódico o Ácido Cítrico Dextrosa (ACD).

El modo de actuación se basa en que el citrato quela el calcio, formando complejos citrato-calcio que alteran los factores de coagulación dependientes del calcio. Disminuye así los niveles de calcio iónico en el circuito extracorpóreo, consiguiendo una anticoagulación regional. Los complejos citrato-calcio son parcialmente eliminados en el líquido efluente, y el resto se metaboliza en el hígado.

Por metabolización hepática el citrato se convierte en bicarbonato en proporción 1:3, quedando libre el calcio. Por ello, debemos monitorizarlo y disponer de una vía venosa central para reponerlo en caso necesario. Hay que tener en cuenta que el citrato también se une al magnesio, por lo que es preciso monitorizar sus niveles.

Para obtener un nivel de anticoagulación adecuado, el flujo de citrato se debe ajustar al flujo de sangre. La concentración objetivo de citrato calculado en el filtro es de 3-5 mmol/L; de esta forma no precisa monitorización aparte y aporta al paciente un buffer fijo. Podemos también medir el calcio postfiltro para monitorizar la anticoagulación, siendo ésta óptima con un nivel de calcio iónico postfiltro de 0.25-0.35 mmol/L. De este modo se aporta un buffer variable al paciente.

Como efectos secundarios derivados del uso de citrato podemos encontrar hipocalcemia, hipomagnesemia, hipernatremia (ya que aporta gran cantidad de sodio), alcalosis metabólica (si la función hepática esta preservada) o acidosis metabólica con anion gap elevado (en caso de disfunción hepática).

El uso de citrato tiene contraindicaciones relativas, entre ellas: disfunción hepática, hipoperfusión tisular, y shock cardiogénico con acidosis láctica significativa (lactato $>2$ $\mathrm{mmol} / \mathrm{L}$ ).

- La AR con heparina consiste en una infusión prefiltro de heparina no fraccionada (HNF) con posterior neutralización de la misma a través de una infusión postfiltro de protamina. Esta modalidad se utiliza poco en la práctica clínica ya que conlleva un alto riesgo que reacciones anafilactoides, así como hipotensión, leucopenia $\mathrm{y}$ trombocitopenia asociada a protamina.

\section{- Anticoagulación sistémica con heparina.}

A pesar de ser una segunda opción (cuando existe alguna contraindicación para el uso de citrato), la HNF es la modalidad de anticoagulación más extendida.

El nivel de anticoagulación se monitoriza mediante el TTPA postfiltro, que debe estar entre 45-55 segundos. Un posible efecto adverso de esta modalidad es la trombocitopenia inducida por heparina (TIH), que se puede desarrollar a los 5-10 días del inicio de la terapia. Esta complicación obliga a la retirada inmediata de la heparina.

La heparina de baso peso molecular (HBPM) tiene limitada experiencia de uso en TCRR. Como potencial ventaja podría presentar un menor riesgo de sangrado y una mayor vida útil del hemofiltro, comparada con HNF. Sin embargo, requiere monitorizar el factor antiXa y no hay evidencia consistente para esta aplicación. 
- Anticoagulación

en situaciones especiales.

Existen otras opciones de anticoagulación indicadas cuando no se puede utilizar citrato ni heparina.

La TIH inmunomediada (tipo 2) es, como se comentó anteriormente, una complicación del uso de heparina. Condiciona un estado protrombótico que requiere anticoagulación sistémica en TCRR. Para ello disponemos de otros agentes anticoagulantes, como argatroban y lepirudina, ambos inhibidores directos de la trombina. Entre estos, se prefiere argatroban ya que presenta metabolismo hepático.

Otra opción más factible es el epoprostenol. Se trata de un análogo sintético de una prostaglandina natural comúnmente denominada prostaciclina, que actúa como un potente inhibidor de la agregación plaquetaria. Se utiliza también como tratamiento de la hipertensión pulmonar arterial, por su efecto vasodilatador. Además, el epoprostenol potencia la actividad anticoagulante de la heparina en aproximadamente un $50 \%$, lo que podría tener un papel como adyuvante en modalidad de anticoagulación sistémica con HNF.

\section{- PROGRAMACIÓN DOSIS EN TRR}

DE

Según la bibliografía se establecen unas dosis estándar para cada modalidad de terapia (tabla 3).

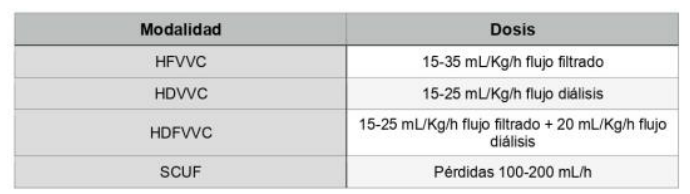

Tabla 3. Dosis recomendadas según tipo de terapia de reemplazo renal.

La evidencia actual sugiere pautar para TCRR una dosis inicial mínima, con un flujo de filtrado de $20-25 \mathrm{ml} / \mathrm{kg} / \mathrm{h}$. Teniendo en cuenta las interrupciones de tratamiento por realización de pruebas o diferentes cuidados, por ejemplo, debemos prescribir $25 \mathrm{ml} / \mathrm{kg} / \mathrm{h}$ en HFVVC. (18)

Se requieren más estudios para determinar con seguridad la dosis óptima de TCRR en pacientes con shock séptico. Una reciente revisión de Cochrane de 2017, concluyó que no hay diferencias de mortalidad utilizando altos flujos (definido por dosis $>50$ $\mathrm{ml} / \mathrm{h}$ ), comparado con terapia estándar.

Actualmente el aclaramiento de solutos, según hallazgos de laboratorio, es el mejor indicador para conocer el rendimiento de las terapias y poder optimizar la dosis de forma individualizada. Así mismo, la extracción de volumen y los balances hídricos deben ser revisados de forma estrecha y ajustar las pérdidas de forma dinámica según la situación clínica del paciente.

\section{- Finalización de TCRR. (19)}

Podemos seguir las siguientes pautas para la finalización de una TDRR:

- Diuresis espontánea mayor de $400 \mathrm{ml} /$ día.

- Disbalances electrolíticos corregidos.

- No acumulación de solutos que necesiten un aclaramiento adicional.

- Balance hídrico estable.

No se recomienda el uso de fármacos diuréticos para tratar de mejorar la recuperación de la función renal, ni durante ni después de la terapia. Por otro lado, el inicio de la diuresis en el paciente no ha demostrado asociar una disminución del tiempo de TCRR. 
- Complicaciones de las
TCRR. (4)

Entre las posibles complicaciones de la TCRR se encuentran las siguientes:

- Complicaciones relacionadas con el catéter venoso de altos flujos. Incluyendo bacteriemias asociadas a catéter.

- Inestabilidad hemodinámica.

- Pérdida sanguínea.

- Consumo de plaquetas.

- Disbalances electrolíticos.

- Hipotermia.

- Embolia aérea. Por ejemplo, por insuficiente purgado de la línea de la máquina de hemofiltración.

- Efectos derivados de la anticoagulación (sangrado). Así como reacciones adversas específicas del anticoagulante utilizado, por ejemplo, trombocitopenia inducida por heparina.

\section{Conclusiones}

Las TCRR son una medida terapéutica cada vez más empleada en las UCI. No obstante, es una técnica compleja que requiere de un manejo multidisciplinar $\mathrm{y}$ de una amplia formación, tanto del personal médico, como del equipo de enfermería y técnicos de cuidados asociados a enfermería.

Las TCRR tienen su principal utilidad en pacientes que presentan inestabilidad hemodinámica, ya que las técnicas intermitentes son, en general, peor toleradas a nivel hemodinámico. Sus indicaciones clásicas son acidosis metabólica severa, uremia severa, sobrecarga de volumen e hiperpotasemia, cuando estas son refractarias a tratamiento médico.

La modalidad escogida de TCRR será variable en función de los solutos que necesitemos eliminar y el estado clínico del paciente.

Las TCRR precisan de la colocación de accesos venosos centrales de altos flujos, siendo la vena yugular derecha de primera elección. Las principales complicaciones de estas técnicas están relacionadas con los accesos venosos (malposición, sangrado, trombosis).

La anticoagulación del sistema es fundamental para el funcionamiento de la terapia. La primera opción es la anticoagulación regional con citrato, seguida de la anticoagulación sistémica con heparina.

Aunque se ha avanzado mucho a la hora de estandarizar las TCRR persisten aspectos que todavía son controvertidos y que son susceptibles de mejoría a medida que se continúe investigando.

\section{Bibliografía}

1. Susantitaphong P, Cruz DN, Cerda J, et al. World incidence of AKI: a metaanalysis. Clin J Am Soc Nephrol 2013;8(9):1482-93). (PubMed)

2. Kidney Disease: Improving Global Outcomes (KDIGO) Acute Kidney Injury Work- group. KDIGO clinical practice guideline for acute kidney injury. Kidney Int 2012; 2:1-138. (HTML)

3. Michael Heung, Lenar Yessayan. Renal Replacement Therapy in Acute Kidney Injury: Controversies and Consensus. Crit Care Clin 33 (2017) 365-378. (PubMed)

4. SS.M. Galvagno Jr. et al. Practical considerations for the dosing and adjustment of continuous renal replacement therapy in the intensive care unit. Journal of Critical Care 2013. (HTML)

5. R. L. Mehta, "Renal-replacement therapy in the critically ill - does timing matter?," New England Journal of Medicine, vol. 375, no. 2, pp. 175176, 2016). (HTML)

6. A. Zarbock, J. A. Kellum, C. Schmidt et al., "Effect of early vs delayed initiation of renal replacement therapy on mortality in critically ill patients 
with acute kidney injury," Jama, vol. 315, no. 20, pp. 2190-2199, 2016. (HTML)

7. S. Gaudry, D. Hajage, F. Schortgen et al., "Initiation strategies for renalreplacement therapy in the intensive care unit," New England Journal of Medicine, vol. 375, no. 2, pp. 122-133, 2016. (NEJM)

8. Fayad AII, Buamscha DG, Ciapponi A. Timing of renal replacement therapy initiation for acute kidney injury. Cochrane Kidney and Transplant Group, editor. Cochrane Database of Systematic Reviews [Internet]. 2018 Dec 18. (HTML)

9. The STARRT-AKI Investigators. Timing of Initiation of RenalReplacement Therapy in Acute Kidney Injury. N Engl J Med. 2020 Jul 16;383(3):240-51. (PubMed)

10. Chávez-Iñiguez JS, Cerdá J. Principios y modalidades en terapia de reemplazo renal continua. GMM. 2019 Jan 29;154(91):799.

11. McCunn M, Reynolds H, Reuter J, McQuillan K, McCourt T, Stein D. Continuous renal replacement therapy in patients following traumatic injury. Int J Artif Organs 2006;29:166-86. (PubMed)

12. Alvarez G, Chrusch C, Hulme T, Posadas-Calleja JG. Renal replacement therapy: a practical update. Can J Anesth/J Can Anesth. 2019 May;66(5):593-604.

13. Ronco, Ricci, De Backer, Kellum, Taccone, Joannidis et all. Renal replacement therapy in acute kidney injury: controversy and consensus. Crit Care. 2015; 19(1): 146 (HTML)

14. Schefold, Haehling, Pschowski, Bender, Berkmann et all. The effect of continuous versus intermittent renal replacement therapy on the outcome of critically ill patients with acute renal failure (CONVINT): a prospective randomized controlled trial. Crit Care 2014 Jan 10;18(1):R11. doi: 10.1186/cc13188. (PubMed)

15. Arif Khwaja. KDIGO Clinical Practice Guidelines for Acute Kidney Injury. Nephron Clin Pract 2012;120:c179c184. (PubMed)

16. Ahmed AR, Obilana A, Lappin D. Renal Replacement Therapy in the Critical Care Setting. Critical Care Research and Practice. 2019 Jul 16;2019:1-11. (HTML)

17. Fayad AI, Buamscha DG, Ciapponi A. Intensity of continuous renal replacement therapy for acute kidney injury. Cochrane Kidney and Transplant Group, editor. Cochrane Database of Systematic Reviews [Internet]. 2016 Oct 4. (ㅂML)

18. Ying Wang, Martin Gallagher et al. Renal replacement therapy intensity for acute kidney injury and recovery to dialysis independence: a systematic review and individual patient data meta-analysis. Nephrol Dial Transplant (2017) 1-8. (ㄴML)

19. Karkar A, Ronco C. Prescription of CRRT: a pathway to optimize therapy. Ann Intesive Care. 2020 Dec;10(1):32. ( $\underline{\text { HTML) }}$

Correspondencia al autor

Carlos Martínez Villar

carlos1mv24@hotmail.com

Facultativo Especialista de Área de Anestesiología y Cuidados Intensivos.

Complejo Hospitalario Universitario de Albacete.

Aceptado para el blog en febrero de 2021 